\title{
Status of XSUSA for Sampling Based Nuclear Data Uncertainty and Sensitivity Analysis
}

\author{
W. Zwermann, L. Gallner, M. Klein, B. Krzykacz-Hausmann, I. Pasichnyk, A. Pautz, K. Velkov \\ Gesellschaft für Anlagen- und Reaktorsicherheit, Forschungszentrum, Boltzmannstrasse 14, \\ Garching, Germany, 85748, Winfried.Zwermann@grs.de
}

\begin{abstract}
In the present contribution, an overview of the sampling based XSUSA method for sensitivity and uncertainty analysis with respect to nuclear data is given. The focus is on recent developments and applications of XSUSA. These applications include calculations for critical assemblies, fuel assembly depletion calculations, and steadystate as well as transient reactor core calculations. The analyses are partially performed in the framework of international benchmark working groups (UACSA - Uncertainty Analyses for Criticality Safety Assessment, UAM - Uncertainty Analysis in Modelling). It is demonstrated that particularly for full-scale reactor calculations the influence of the nuclear data uncertainties on the results can be substantial. For instance, for the radial fission rate distributions of mixed $\mathrm{UO}_{2} / \mathrm{MOX}$ light water reactor cores, the $2 \sigma$ uncertainties in the core centre and periphery can reach values exceeding $10 \%$. For a fast transient, the resulting time behaviour of the reactor power was covered by a wide uncertainty band. Overall, the results confirm the necessity of adding systematic uncertainty analyses to best-estimate reactor calculations.
\end{abstract}

\section{Introduction}

Evaluated nuclear data files are continuously being improved. Recently, the European library was updated to JEFF-3.1.1 [1], the American library to ENDF/B VII.1 [2], and the Japanese library to JENDL-4.0 [3]. These library improvements are performed on the basis of the newest evaluations of differential experiments. Nevertheless, their precision is limited by the uncertainties of the underlying measurements and theoretical parameters. There is an increasing effort to improve the amount and quality of the covariance files accompanying the major data libraries. For now, a rather complete set of covariance data is provided in multi-group format with the SCALE 6 system [4]. In the past, most uncertainty investigations with nuclear covariance data, as performed, e.g., with TSUNAMI [5] or SUSD3D [6], were based on first order perturbation theory, and primarily consider the multiplication factors and other integral quantities. With increasing computer power, another approach has become possible, namely random sampling of nuclear data, as implemented in MCNPACAB [7], NUDUNA [8], TMC [9], and XSUSA [10]. Meanwhile, the GRS code package XSUSA ("Cross Section Uncertainty and Sensitivity Analysis") has been used for a wide variety of calculations for fissionable systems, including neutron transport calculations for pin cells, fuel assemblies, critical experiments, and full-scale reactor calculations, fuel assembly depletion calculations, as well as coupled neutron transport/thermo-hydraulic steady-state and transient reactor core calculations. 


\section{The XSUSA Method}

Within the sampling based GRS method implemented in the code package SUSA ("Software for Uncertainty and Sensitivity Analysis”) [11], many calculations for the problem under consideration are performed with varied input data. The variations of the input data are generated randomly from the given probability distributions of the parameters including possible correlations between them. After performing the complete series of calculations, the output quantities of interest are statistically analysed, and their uncertainty ranges and sensitivities to the input parameters are determined.

Originally, the GRS method has been mainly applied to problems with a limited number of parameters and only few correlations between them, such as thermo-hydraulic or technological parameters. However, in the case of its application to the nuclear data uncertainties implemented in XSUSA, various reactions of various nuclides have to be considered. Using the nuclear covariance data from the SCALE 6 code package, 44 uncertain parameters for each nuclide and reaction corresponding to the 44-group structure are analysed, resulting in a huge overall number of uncertain parameters. Moreover, a large amount of correlations between the energy group data of each nuclide/reaction combination have to be taken into account, and also cross correlations between data of different reactions and nuclides.

The nuclear data covariance matrices only contain the relative variance and covariance values of the nuclear data, i.e. the second moments of the distributions; the types of the distributions are not explicitly known and assumed to be Gaussian.

To use the GRS method with nuclear covariance data, the ENDF/B-VII based 238-group library of SCALE 6 is either used as is, or pre-collapsed to the 44-group structure of the covariance data using a flux spectrum typical for the system under consideration, which can be advantageous when doing full core calculations which require the handling of large amounts of data for the different fuel assemblies in various burn-up states. With the original or the collapsed master library, all necessary spectral calculations are performed, using the Bondarenko method implemented in the BONAMI module for the unresolved resonance regions, and performing 1-D transport calculations by the CENTRM module with continuous energy data in the resolved resonance region. The resulting data libraries are modified according to the uncertainty information in the covariance matrices for each nuclide/reaction combination considered. After doing so, it has to be assured that the cross section set is entirely consistent, i.e. that sum rules are fulfilled and that 2-D cross sections (e.g. scattering matrices) are compatible with their 1-D counterparts.

\section{Applications}

In this Section, uncertainty and sensitivity analysis results are presented for a variety of neutron transport applications.

\subsection{Critical Assembly Calculations}

In order to apply XSUSA to systems with different spectral conditions, Uranium and Plutonium systems are chosen, with fast and thermal spectra. Three of them are described in the International Handbook of Evaluated Criticality Safety Benchmark Experiments [12]: The bare metallic Uranium sphere GODIVA (in the nomenclature of the Handbook HEU-MET-FAST-001), the bare metallic Plutonium sphere 239Pu JEZEBEL (PU-MET-FAST-001), and one of the P-11 series of bare spheres of Plutonium nitrate solutions (PU-SOL-THERM-011, Case 16-1). KRITZ-2:13 is a light water moderated quadratic array of Uranium fuel pins, described in the International Handbook of Evaluated Reactor Physics Benchmark Experiments [13] as KRITZ-LWR-RESR-003. Due to their spherical shapes, GODIVA, JEZEBEL, and the P-11 sample were calculated with the 1-D deterministic XSDRN code as transport solver, while for KRITZ-2:13, the 3-D Monte Carlo code KENO-Va was used, both with XSUSA and TSUNAMI. Both XSDRN and KENO-Va are part of 
the SCALE 6 system. In the XSUSA analysis, 1,000 samples were evaluated. The multiplication factor uncertainties from both the approaches agree very well.

Table 1. Multiplication factors and corresponding uncertainties due to nuclear data covariances obtained from XSUSA and TSUNAMI with sampled nuclear data.

\begin{tabular}{|l|c|c|c|c|}
\hline & GODIVA & JEZEBEL & KRITZ-2:13 & P-11, 16-1 \\
\hline Multiplication factor & 1.00016 & 1.00035 & 0.99644 & 1.01042 \\
\hline Uncertainty from XSUSA & $1.063 \%$ & $1.418 \%$ & $0.512 \%$ & $1.506 \%$ \\
\hline Uncertainty from TSUNAMI & $1.069 \%$ & $1.389 \%$ & $0.529 \%$ & $1.475 \%$ \\
\hline
\end{tabular}

\subsection{Steady-State Core Calculations}

The XSUSA method has been applied to a variety of full-scale reactor core calculations; as an example, a 2-D calculation for the uncontrolled hot zero-power state of a PWR mixed core specified within an international OECD/NEA benchmark [14] is chosen. Figure 1 displays the XSUSA uncertainty results (relative $1 \sigma$ values) in the radial power distribution from the reference Monte Carlo calculations with KENO (left side). In addition, nodal calculations were performed with the GRS diffusion code QUABOX-CUBBOX with two-group fuel assembly homogenized cross sections obtained with the lattice code NEWT from SCALE 6. The corresponding results are given on the right side of Fig. 1.
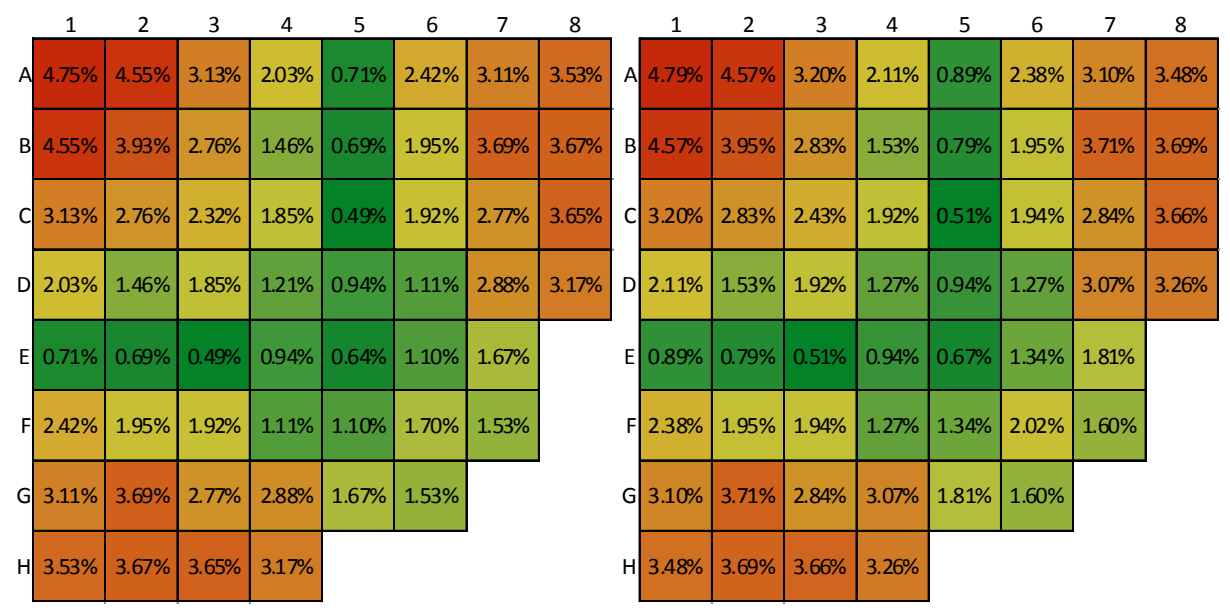

Fig. 1. Uncertainties of the radial fuel assembly power distribution of a mixed PWR core. Left: XSUSA/KENO; right: XSUSA/NEWT/QUABOX-CUBBOX

The resulting $1 \sigma$ uncertainty is almost $5 \%$ for the power in the central fuel assembly; depending on the core layout, even larger uncertainties have been observed in full-scale core calculations. In addition, it is remarkable that the uncertainty distributions of both calculations are practically identical, demonstrating that the output uncertainties are hardly influenced by the additional calculation steps performed by the lattice code.

\subsection{Time-dependent Core Calculations}

For the same benchmark, an exercise for a transient was specified, namely the ejection of one control rod. This transient was analysed with the coupled GRS code system QUABOX-CUBBOX/ ATHLET. Figure 2 shows a substantial uncertainty band for the evolution of the reactor power, 
ranging from practically no power excursion at all to a power peak of several times the nominal full reactor power.

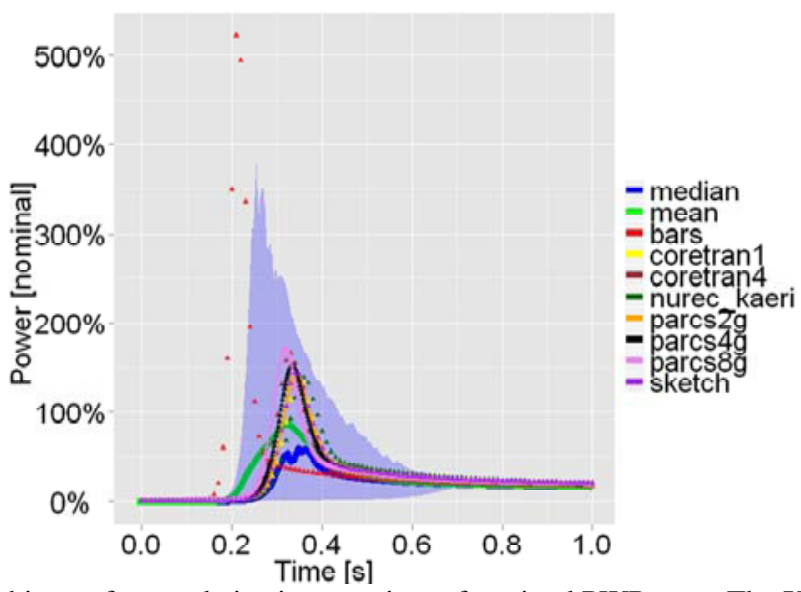

Fig. 2. Core power history for a rod ejection transient of a mixed PWR core. The XSUSA/QUABOXCUBBOX/ATHLET uncertainty band is compared with the results of the benchmark participants.

\subsection{Burn-Up Calculations}

XSUSA is also applied for determining uncertainties in the relevant output quantities of pin cell and fuel assembly burn-up calculations, such as nuclide inventories and few-group cross sections for subsequent core simulations. Figure 3 gives an example for the isotope uncertainties arising in a $\mathrm{UO}_{2}$ fuel pin during five years of irradiation and five years of cooling time. The calculations were performed with the SCALE 6 depletion sequence TRITON.

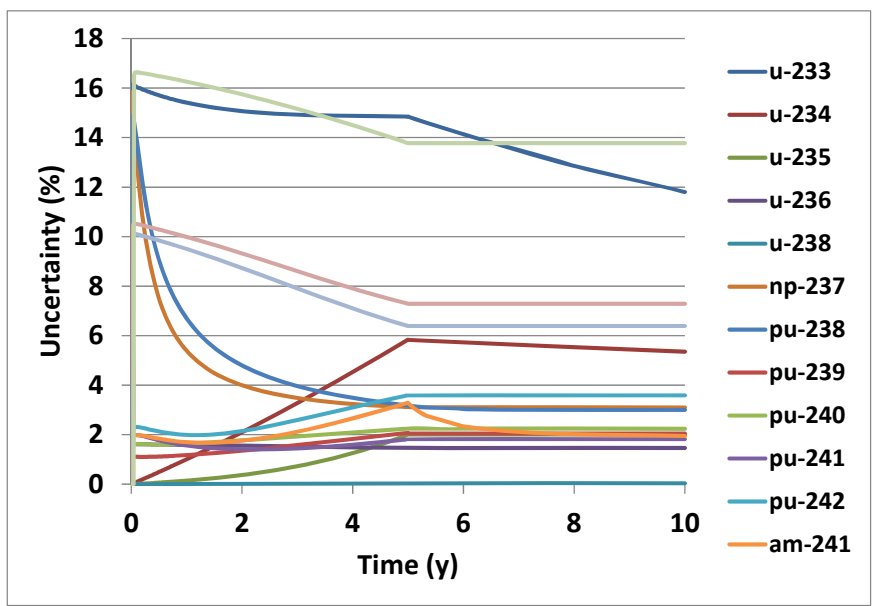

Fig. 3. Nuclide inventory uncertainty in a $\mathrm{UO}_{2}$ pin cell burn-up calculation.

\section{Conclusions and Outlook}

The sampling based XSUSA cross section uncertainty and sensitivity analysis sequence has reached a mature state and is being applied to a variety of neutron transport calculations, namely criticality, depletion, lattice, and full core calculations, using both Monte Carlo and deterministic transport 
methods. So far, the analyses were restricted to neutron cross sections as uncertain parameters. This is currently being extended to additional quantities relevant for burn-up calculations, namely fission yield and decay data. Another extension is being performed concerning the inclusion of delayed neutron multiplicity uncertainties, which may be important for the time-dependent behaviour of fissionable systems. When using Monte Carlo codes as transport solvers, methods are under investigation to drastically reduce the number of neutron histories without losing much information on the output uncertainties.

In conclusion, it is desirable and feasible to routinely accompany all parts of reactor calculations by uncertainty and sensitivity analyses, along with aiming for evaluated nuclear data of highest quality, as well as reliable and complete nuclear data uncertainty information.

This work is supported by the German Federal Ministry of Economics and Technology.

\section{References}

1. A. Santamarina et al., “The JEFF-3.1.1 Nuclear Data Library,” JEFF Report 22, NEA No. 6807 (2009).

2. M. Herman, "Development of ENDF/B-VII.1 and Its Covariance Components", Journal of the Korean Physical Society, 59, pp.1034-1039 (2011).

3. K. Shibata et al., "JENDL-4.0: A New Library for Innovative Nuclear Energy Systems,” Journal of the Korean Physical Society, 59, pp.1046-1051 (2011).

4. "SCALE: A Modular Code System for Performing Standardized Computer Analyses for Licensing Evaluation, Version 6“, ORNL/TM-2005/39 (2009).

5. B.T. Rearden and D.E. Mueller, "Uncertainty Quantification Techniques of SCALE/ TSUNAMI”, Trans. Am. Nucl. Soc., 104, pp.371-373 (2011).

6. I. Kodeli, "The SUSD3D Code for Cross-Section Sensitivity and Uncertainty Analysis - Recent Development,” Trans. Am. Nucl. Soc., 104, pp.791-793 (2011).

7. N. García-Herranz, O. Cabellos, J. Sanz, J. Juan, and J. C. Kuijper, "Propagation of Statistical and Nuclear Data Uncertainties in Monte Carlo Burnup Calculations,” Annals of Nucl. Energy, 35, pp.714-730 (2008).

8. O. Buss, A. Hoefer, and J.C. Neuber, "NUDUNA - Nuclear Data Uncertainty Analysis,” Proc. International Conference on Nuclear Criticality (ICNC 2011), Edinburgh, Scotland, Sep. 19-22, 2011, on CD-ROM (2011).

9. A. J. Koning and D. Rochman, "Towards Sustainable Nuclear Energy: Putting Nuclear Physics to Work,” Annals of Nucl. Energy, 35, pp.2024-2030 (2008).

10. W. Zwermann, B. Krzykacz-Hausmann, L. Gallner, A. Pautz, "Influence of Nuclear Covariance Data on Reactor Core Calculations," Second International Workshop on Nuclear Data Evaluation for Reactor Applications (WONDER 2009), Cadarache, France, 29 Sep. - 2 Oct., 2009, pp.99-104 (2009).

11. B. Krzykacz, E. Hofer, M. Kloos, "A Software System for Probabilistic Uncertainty and Sensitivity Analysis of Results from Computer Models," Proc. International Conference on Probabilistic Safety Assessment and Management (PSAM-II), San Diego, Ca., USA, (1994).

12. "International Handbook of Evaluated Criticality Safety Benchmark Experiments", September 2010 Edition, available on DVD-ROM, NEA/NSC/DOC(95)03.

13. "International Handbook of Evaluated Reactor Physics Benchmark Experiments", March 2010 Edition, available on DVD-ROM, NEA/NSC/DOC(2006)1.

14. T. Kozlowski, T. J. Downar, "The PWR MOX/UO2 Core Transient Benchmark, Final Report”, NEA/NSC/DOC(2006)20. 